\title{
Big five traits and perfectionism are risk factors for nonsuicidal cutting
}

\author{
Holly M. Miskey, Robert W. Hill, Timothy J. Huelsman \\ Dept. of Psychology, Appalachian State University, Boone, USA
}

Email address:

hmmiskey@uncg.edu (H. M. Miskey),hillrw@appstate.edu (R. W. Hill)

\section{To cite this article:}

Holly M. Miskey, Robert W. Hill, Timothy J. Huelsman. Big Five Traits and Perfectionism Are Risk Factors for Nonsuicidal Cutting. Psychology and Behavioral Sciences. Vol. 1, No. 1, 2012, pp. 1-7. doi: 10.11648/j.pbs.20120101.11

\begin{abstract}
This investigation assessed the role of the Big Five dimensions of personality and perfectionism in predicting nonsuicidal cutting in a sample of undergraduate students. Of 292 students, 50 (17\%) endorsed cutting behavior. Duration of cutting was associated with Openness, and Introversion, as well as perfectionistic rumination. Frequency of cutting was associated with Conscientiousness, Agreeableness, low Neuroticism (accounting for about 20\% of the variance), as well as perfectionistic rumination, organization, and low concern over mistakes (accounting for $31 \%$ of the variance). These findings are discussed in the context of previous research investigations of risk factors for nonsuicidal self-injury (NSSI) that are convergent, and sometimes discrepant with these data. The findings suggest that more research into personality traits and methods of NSSI is warranted.
\end{abstract}

Keywords: Self-injury; Personality; Perfectionism; Risk factors; Cutting

\section{Introduction}

Non-suicidal self-injury (NSSI) can be defined as "the direct, deliberate destruction or alteration of one's own body without conscious suicidal intent" [1]. This definition excludes overdoses, substance use, and swallowing of poisons or objects as well as suicide attempts, regardless of ambivalence level. The prevalence of NSSI among both clinical and non-clinical samples is startling. Reports vary from a six-month prevalence rate of $4 \%$ of the general adult population $[2,3]$ to $35 \%$ in a clinical population [4]. Previous literature demonstrates a higher prevalence of self-injurious acts in an undergraduate population than in the general population ranging from a lifetime prevalence of $17 \%$ [5] to $38 \%$ [6].

The most common form of NSSI in clinical samples is cutting oneself [2]. In a sample of high school adolescents, $14 \%$ were found to have cut [7]. Samples of college students have found similar rates [8,9]. Despite these high prevalence rates, few studies have attempted to focus specifically on individuals who report nonsuicidal cutting behavior. This may be due to the typical practice of researchers studying multiple methods of NSSI. Investigations have discovered $62 \%$ [10] to $71 \%$ [5] of undergraduates who engaged in NSSI used more than one method. As a result, researchers are likely unable to find an adequate sample of participants who only cut. However, the high prevalence of nonsuicidal cutting indicates the need for more information on this subgroup. The purpose of the current study is to address this knowledge gap by exploring risk factors associated with nonsuicidal cutting in undergraduates.

\section{Research on NSSI}

\subsection{NSSI and the Big Five}

As there is no research specific to nonsuicidal cutting, we turned to the larger literature on risk factors for NSSI in general. Well documented risk factors include dissociation [11], emotional dysregulation [12], and psychopathology such as Borderline Personality Disorder, Major Depressive Disorder, Posttraumatic Stress Disorder (PTSD), and Depersonalization Disorder [1,4]. Recent research suggests sexual abuse is not a risk factor for NSSI; rather it is the resulting PTSD symptomatology [13]. These internal factors may be related to long-standing personality traits; however, few studies have considered personality traits of individuals who self-injure, and available studies often offer contradictory findings. Using the Five Factor Model, clinical samples of those practicing NSSI have demonstrated significantly lower levels of Extraversion [14, 15] and higher levels of Neuroticism [14] while a sample of prisoners reported higher levels of Extraversion [16]. Studies of NSSI using 
undergraduate samples have found higher levels of Neuroticism and Openness to experience $[8,17]$ but have differed on the remaining factors. Brown [8] discovered lower levels of Agreeableness and Conscientiousness in undergraduates reporting NSSI, with no differences in Extraversion while Goldstein and colleagues [17] reported no association with Extraversion, Agreeableness or Conscientiousness. Recently, MacLaren and Best [18] found NSSI was significantly associated with facets of Neuroticism, Agreeableness and Conscientiousness, but not Extraversion.

The variability in findings for NSSI and Big Five variables may be a consequence of differing samples, inconsistent measures of Big Five traits, and perhaps inconsistent means of assessing NSSI. It is also possible different methods of NSSI may be associated with different personality profiles. MacLaren and Best [18] observed the longstanding association between NSSI, particularly cutting behavior, and Borderline Personality Disorder and point out the growing literature associating personality disorder pathology with Big Five traits as a tool for diagnosis $[19,20]$. An investigation exploring the Big Five and nonsuicidal cutting not only contributes to a void in the research, but is also consistent with the shift in psychology toward dimensional conceptualization of diagnoses. Based on available research, we predicted cutting behavior would be associated with higher levels of Neuroticism and Openness. Due to the inconsistent findings with the remaining factors, no hypotheses were posited.

\subsection{NSSI and Perfectionism}

Perfectionism has long been associated with psychological distress [21], and a modest literature links perfectionism to NSSI. Recently, Hoff and Muehlenkamp [22] found that the Parental Criticism, Concern over Mistakes, and Organization scales from the Multidimensional Perfectionism Inventory [23] were significantly and positively associated with NSSI in an undergraduate sample. In other words, undergraduates endorsing NSSI placed more emphasis on parental expectations and evaluations, were more worried about making errors, and tended to be more orderly. Rumination is also considered to be a facet of perfectionism. Using a rumination measure with subscales for brooding and assessment, Hoff and Muehlenkamp [22] found students who self-injured were higher on both scales than controls, and regression analyses indicated brooding, but not reflection, accounted for unique variance when predicting self-injury status. Brooding rumination includes thinking about depression and anxiety related symptoms, and the possible causes and consequences of those symptoms [24].

A study of Scottish adolescents (mean age 15.2 years) defining self-injury as deliberate harm to self or overdosing reported significant positive associations between self-injury and self-oriented (intense motivation for perfection and high expectations for the self) and socially-prescribed (belief others hold unrealistic expectations for the self) perfectionism [25]. The results from these two studies suggest a relationship between perfectionism and
NSSI; however, nothing is known of how perfectionism may relate to specific methods of NSSI. Additionally, factor analytic studies suggest that perfectionism is best described through two factors: Personal Standards Perfectionism, which involves having high achievement standards, being organized and planful, and having high standards for others, and Self-Evaluative Perfectionism, which involves being concerned about making mistakes, ruminating about performance, and concern for the approval of others for performance [21]. We anticipated that Self-Evaluative Perfectionism would be particularly associated with nonsuicidal cutting. Thus the current investigation provides data on the relationship between cutting and personality traits, specifically the Big Five traits and perfectionism, in a non-clinical sample.

\section{Method}

\subsection{Participants and Procedure}

The investigation was reviewed and approved by the university Institutional Review Board. Undergraduate students were solicited from introductory psychology courses at a Southeastern, medium sized, American university and offered course credit. A total of 292 undergraduate students completed questionnaire packets in groups. The mean age of the total sample was $19.10(\mathrm{SD}=2.05)$ with a majority identifying as Caucasian (88.7\%), followed by African American (5.5\%), Hispanic (3.1\%), Multiracial or Native American (2.4\%), and Asian (.3\%). Women comprised 62.3\% of the sample and the majority described themselves as single (97.3\%). Of the 292 participants, 50 (17\%) endorsed cutting behaviors which was chosen as the target NSSI behavior for subsequent analyses for determining age of onset and duration of NSSI.

The 50 participants who endorsed cutting included 40 women and 10 men ranging in age from 16 to 38 years $(\mathrm{M}=$ 19.72, SD = 3.43). All participants reported having intentionally cut themselves at least once, but the number of episodes ranged from 1 to more than 1100 times (Median = $6.50, \mathrm{M}=44.46, \mathrm{SD}=169.08$; only two participants reported cutting themselves more than 100 times).

The participants' ages when they first cut ranged from 11 to 18 years $(\mathrm{M}=14.54, \mathrm{SD}=1.73)$. More than half of the participants $(60 \%)$ reported exposure to NSSI prior to engagement, but less than half (38\%) reported that knowing other people who did it "played a role" in their behavior. The most recent incident of cutting occurred from 8 days to 19 years ago $($ Median $=3.00$ years, $\mathrm{M}=2.68$ years, $\mathrm{SD}=2.96$ years). Most participants reported previous attempts to stop (78\%). Only a small percentage (8\%) reported seeking treatment for injuries resulting from cutting, though a much larger percentage (38\%) reported injuries serious enough that they "should have sought medical attention." The majority of the participants (84\%) engaged in additional forms of NSSI, most commonly scratching causing scarring or bleeding (42\%) and burning (28\%). 
Approximately half of all participants who reported cutting $(48 \%)$ reported having been diagnosed with a psychological disorder by a psychologist $(58 \%)$, psychiatrist $(21 \%)$, or family physician $(17 \%)$. The most frequent diagnoses were depression (26\%) and ADHD (16\%). About a quarter of participants who cut $(28 \%)$ reported more than one diagnosis. Almost half of all participants who cut (48\%) reported experiencing unwanted sexual encounters prior to age 18 including unwanted kissing or hugging (38\%), unwelcome touching of their sex parts $(30 \%)$, unwanted intercourse $(20 \%)$, and unwanted attempted intercourse (18\%). Numerous participants who cut $(38 \%)$ reported some form of physical abuse (i.e., being hit, kicked, choked, slapped, burned, or beaten).

\subsection{Measures}

\subsubsection{Deliberate Self-Harm Inventory (DSHI)}

The DSHI [26] asks users to report self-injurious practices. Seventeen yes or no questions query different methods of self-injury. An answer of "yes" prompts the user to provide additional information on age of onset, number of incidents, most recent incident, length of time engaging in the behavior, and whether medical treatment was ever required. A total of 16 different types of self-injury are surveyed on the questionnaire. This investigation utilized frequency defined as the total number of cutting incidents over the lifetime, age of onset for first episode of cutting, and duration defined as the total number of months participants reported engaging in cutting. Construct validity has been demonstrated between a dichotomous variable from the DSHI and other measures of NSSI, and convergent validity has been demonstrated for both the dichotomous variable and frequency of DSH [26].

\subsubsection{International Personality Item Pool (IPIP) Big 5}

The IPIP Big 5 measure [27] is a measure of the Big Five personality traits as represented in the NEO-PI-R. On all subscales, items are scored on a 5-point Likert-type scale ranging from 1 "very inaccurate" to 5 "very accurate." The IPIP offers the choice of a 10-item scale, a 20-item scale, or a 60 -item scale comprised of 6 facet subscales (10 items each) for each personality trait. For this investigation, the IPIP 60-item Neuroticism and Extraversion scales were used, and the 20-item Openness, Agreeableness, and Conscientiousness scales were used. The scales have been shown to be highly correlated with the NEO-PI-R scales ( $r$ $=.79$ to .86$)$ and demonstrate good internal consistency (mean $\alpha=.89$ ) [27].

\subsubsection{The Perfectionism Inventory (PI)}

The PI [28] is an empirically derived self-report measure of perfectionism. The PI consists of 59 items comprising eight scales with coefficient alphas ranging from .83 to .91. The Conscientious Perfectionism composite score is derived by summing four scales (Organization, Striving for Excellence, Planfulness, and High Standards for Others) and the Self-Evaluative Perfectionism composite score is derived by summing the remaining four scales (Concern over Mistakes, Need for Approval, Rumination, and Perceived Parental Pressure). In this investigation, the Conscientious Perfectionism composite was used to assess perfectionistic strivings, and the Self-Evaluative Perfectionism composite was used to assess perfectionistic concerns. The construct validity of PI indices, including exploratory and confirmatory factor analyses, and evidence describing the success of the PI in accounting for variance beyond that accounted for by other multidimensional perfectionism measures when predicting various symptom indices was described by Hill and colleagues [28].

\section{Results}

\subsection{Descriptive Statistics}

Means, standard deviations, and internal consistency reliability coefficients for all study variables (see Table 1) are consistent with those reported previously [27, 28]. Cutting duration was positively correlated with Rumination and Openness, and negatively correlated with Extraversion. Cutting frequency was positively correlated with Conscientiousness, Agreeableness, and Extraversion, and negatively correlated with Neuroticism. Cutting onset age was positively correlated with Neuroticism, Concern over Mistakes, and Need for Approval, and negatively correlated with Openness. Onset age was also negatively correlated with frequency. We performed independent samples t-tests on all criterion variables (duration, frequency, age at onset), all Five Factor predictors, and all perfectionism predictors to examine the differences between male and female participants, participants with and without psychological diagnoses, participants reporting presence and absence of physical abuse, and between those reporting presence and absence of sexual abuse. Bonferroni adjustments to alpha levels were applied to partially control for type 1 error. The traditional .05 alpha was divided by three when examining differences on the criterion variables, resulting in an alpha of .017. Using the same rationale, tests of the Big Five factors employed an alpha of .010, and analyses for the eight perfectionism scales had an alpha of. 006. Even using these rather liberal standards (given the 64 pairwise comparisons made), only three statistically significant differences emerged. Female participants $(\mathrm{M}=36.88)$ were higher than male participants $(\mathrm{M}=30.50)$ on Agreeableness $(\mathrm{t}=-2.79$, $\mathrm{p}=.008)$ and Need for Approval $(\mathrm{t}=-3.19, \mathrm{p}=.002 ; \mathrm{M}=$ 3.61 and $\mathrm{M}=2.56$, respectively), and participants who reported physical abuse $(\mathrm{M}=3.85)$ were higher than those reporting no abuse $(M=2.97)$ on Planfulness $(t=-2.93, p$ $=.006$ ). Because of the small number of differences between these various groups, all participants were combined for subsequent analyses. 
Table 1. Correlations and descriptive statistics for study variables.

\begin{tabular}{|c|c|c|c|c|c|c|}
\hline & $r$ with Duration & $r$ with Frequency & $r$ with Onset & $M$ & $S D$ & $\mathbf{A}$ \\
\hline Cutting Duration (months) & -- & & & 30.70 & 49.10 & -- \\
\hline Cutting Frequency & .10 & -- & & 44.46 & 169.08 & -- \\
\hline Cutting Age at Onset (years) & -.21 & $-.35 * *$ & -- & 14.54 & 1.73 & -- \\
\hline Neuroticism & .23 & $-.33 * *$ & $.35 * *$ & 185.80 & 37.45 & .95 \\
\hline Extraversion & $-.30 *$ & $.25^{*}$ & -.05 & 204.80 & 27.91 & .92 \\
\hline Openness & $.25^{*}$ & .09 & $-.26^{*}$ & 40.64 & 3.24 & .77 \\
\hline Agreeableness & -.22 & $.26^{*}$ & -.17 & 35.30 & 6.89 & .84 \\
\hline Conscientiousness & .06 & $.28^{*}$ & -.02 & 64.28 & 12.31 & .90 \\
\hline Concern over Mistakes & .20 & -.20 & $.25 *$ & 2.86 & .94 & .90 \\
\hline High Standards for Others & .05 & .03 & .13 & 2.79 & .87 & .84 \\
\hline Need for Approval & .19 & -.11 & $.24 *$ & 3.40 & 1.01 & .91 \\
\hline Organization & .08 & .23 & .18 & 2.83 & 1.11 & .94 \\
\hline Perceived Parental Pressure & .02 & .08 & .06 & 3.40 & 1.16 & .95 \\
\hline Rumination & $.29 *$ & .00 & .11 & 3.22 & .95 & .89 \\
\hline Striving for Excellence & .14 & -.02 & -.02 & 2.98 & .98 & .88 \\
\hline Planfulness & .22 & .19 & -.11 & 3.39 & 1.01 & .93 \\
\hline
\end{tabular}

Note: $N=50 ; r=$ Pearson correlation; $\alpha=$ Cronbach's alpha, $* p<.05, * * p<.01$.

\subsection{Multiple Regression Analyses}

A series of six separate standard multiple regressions that regressed one of three criteria (duration of cutting, frequency of cutting, or age at onset) on both the Five Factor variables (see Table 2) and the eight perfectionism scales (see Table 3) were performed.1 The regression predicting duration from the Five Factor variables was statistically significant $(\mathrm{F}=$ $2.762, \mathrm{p}=.030$ ), accounting for approximately $24 \%$ of the variance, with Openness making a statistically significant unique contribution to the criterion. The regression predicting frequency from the Five Factor variables failed to reach the traditional criterion for statistical significance $(\mathrm{F}=$ $2.214, \mathrm{p}=.067$ ), but accounted for approximately $20 \%$ of the variance with Conscientiousness making a unique contribution to the criterion that was near the traditional standard for statistical significance. Similarly, the regression

\footnotetext{
${ }^{1}$ While it was not our intention to test whether personality could predict whether or not a participant would engage in cutting behaviors, we did perform two logistic regressions (one for the IPIP scales, another for the PI scales) to test this possibility. For personality (IPIP), the full model was statistically significant, $\chi^{2}$ $(5, \mathrm{~N}=292)=53.92, p<.001$, but classification was unimpressive with $91.8 \%$ of non-cutters correctly predicted but only $38.1 \%$ of cutters correctly predicted. According to the Wald criterion, Neuroticism, $\chi^{2}(1)=7.06, p=.008$, and Openness, $\chi^{2}(1)=26.01, p<.001$, predicted cutting status. For perfectionism, the full model was not statistically significant, $\chi^{2}(7, \mathrm{~N}=292)=12.43, p=.087$, indicating that as a set, the perfectionism scales did not predict cutting status.
}

predicting age of onset from the Five Factor variables failed to reach the traditional criterion for statistical significance $(\mathrm{F}$ $=2.106, \mathrm{p}=.083)$, but accounted for approximately $19 \%$ of the variance with Neuroticism making a unique contribution to the criterion that was near the traditional standard for statistical significance. Similarly, the regression predicting age of onset from the Five Factor variables failed to reach the traditional criterion for statistical significance $(\mathrm{F}=2.106$ $\mathrm{p}=.083$ ), but accounted for approximately $19 \%$ of the variance with Neuroticism making a unique contribution to the criterion that was near the traditional standard for statistical significance.

Table 2. Cutting outcomes regressed on Five Factor Model personality variables.

\begin{tabular}{llll}
\hline & Duration & Frequency & Age at Onset \\
\hline Neuroticism $(\beta)$ & .131 & -.235 & $.372 *$ \\
Extraversion $(\beta)$ & -.236 & .086 & .172 \\
Openness $(\beta)$ & $.338^{*}$ & .057 & -.217 \\
Agreeableness $(\beta)$ & -.206 & .126 & -.085 \\
Conscientiousness $(\beta)$ & .162 & $.237 \dagger$ & -.008 \\
$R^{2}$ & .239 & .203 & .193 \\
$F$ & 2.762 & 2.214 & 2.106 \\
$P$ & .030 & .067 & .083 \\
\hline
\end{tabular}

Note: $d f=5,44 ; * p<.05 ; \not p<.10$. 
Table 3. Cutting outcomes regressed on perfectionism variables.

\begin{tabular}{llll}
\hline & Duration & Frequency & Age at Onset \\
\hline Concern over Mistakes $(\beta)$ & .011 & $-.784^{* *}$ & .272 \\
High Standards for Others $(\beta)$ & .043 & .108 & .250 \\
Need for Approval $(\beta)$ & -.210 & -.302 & .295 \\
Organization $(\beta)$ & .019 & $.452^{*}$ & $.336 \dagger$ \\
Perceived Parental & -.165 & .255 & -.034 \\
Pressure $(\beta)$ & & & \\
Rumination $(\beta)$ & .553 & $.809^{*}$ & -.080 \\
Striving for Excellence $(\beta)$ & -.123 & -.215 & -.328 \\
Planfulness $(\beta)$ & .101 & -.026 & -.371 \\
$R^{2}$ & .129 & .312 & .271 \\
$F$ & .760 & 2.324 & 1.902 \\
$P$ & .639 & .037 & .086 \\
\hline
\end{tabular}

Note: $d f=8,41 ;{ }^{*} p<.05 ; * * p<.01 ; \dagger p<.10$.

The regression predicting cutting duration from the perfectionism variables was not statistically significant $(F$ $=.760, p=.639$ ), accounting for about $13 \%$ of the variance. The regression predicting cutting frequency from the perfectionism variables was statistically significant $(F=2.324$, $p=.037$ ), accounting for approximately $31 \%$ of the variance, with (in descending order of importance) Rumination, Concern over Mistakes (negatively weighted), and Organization making statistically significant unique contributions to the criterion. The regression predicting onset of cutting from the perfectionism variables failed to reach the traditional criterion for statistical significance $(F=1.902, p$ $=.086$ ), but accounted for approximately $27 \%$ of the variance with Organization making a unique contribution to the criterion that was near the traditional standard for statistical significance.

\section{Discussion}

The purpose of this investigation was to assess risk factors associated with nonsuicidal cutting, specifically perfectionism and personality characteristics. Consistent with previous samples of undergraduates, for example Brown [8], the current sample reported cutting as the most common form of self-injury with a prevalence of $17 \%$. The majority ( $84 \%$ ) of those who endorsed cutting also typically engaged in other diverse forms of self-injury, again consistent with previous research [e.g., 5].

\subsection{Cutting and the Big Five}

Regression analyses found the Big Five accounted for $20 \%$ of the variance in frequency of nonsuicidal cutting, which approached but did not meet the traditional standards of statistical significance $(p=.067)$. Frequency was correlated negatively with Neuroticism and positively with Agreea- bleness, which includes characteristics such as trust, compliance, pleasantness, accommodation, modesty, and concern for social harmony [29]. Additionally, frequency was associated with Conscientious traits, typically described as being competent, orderly, dutiful, deliberate, self-disciplined and achievement oriented [29]. These results run contrary to both Brown's [8] and Goldstein and colleagues' [17] findings; however Brown [8] considered multiple methods of NSSI while Goldstein and colleagues' [17] definition of NSSI was even broader, incorporating substance abuse and instances of intent to inflict damage regardless of the outcome (e.g., placing self in dangerous situations). These varied outcomes may partly reflect different definitions of NSSI as the current study focused on cutting and suggests the possibility of different personality profiles for different methods of self-injury. This investigation suggested an interestingly positive view of personality features associated with cutting frequency: those who engage in more cutting have personalities marked by less neurotic distress and more agreeable and conscientious features.

The Five Factors also accounted for $24 \%$ of the variance predicting duration of cutting with Openness providing the only unique contribution. The length of time participants cut was predicted by Openness, which has been described as a preference for variety, intellectual curiosity, imagination, attention to feelings, and a less conventional approach to life [29]. Additionally, duration was associated with higher levels of introversion. Interestingly, duration and frequency of cutting were not correlated $(\mathrm{r}=.10)$ in this sample, and the two variables had inverse associations with Extraversion. Apparently, these two indices of self-injury independently assess facets of cutting: the length of time one cut was not related to the number of times one cut. Extraverts cut more, and introverts cut longer.

Additionally, cutting onset age was negatively correlated with frequency. The younger one started, cutting the more one cut. However, onset age was not significantly correlated with duration of cutting. The correlation between onset age and frequency may be a reflection of stunted coping skill development: individuals who begin cutting earlier may rely more heavily on this behavior to modulate emotion or regulate stress rather than developing other coping strategies. Consistent with this interpretation, age of onset was also positively correlated with Neuroticism, and negatively with Openness, suggesting the older one starts cutting the more neurotic personality traits are likely, and the less open to new experiences. Regression analyses found the Big Five accounting for $19 \%$ of the variance in age of onset, which approached but did not meet the traditional standards of statistical significance $(\mathrm{p}=.083)$.

\subsection{Cutting and Perfectionism}

The perfectionism scales accounted for $31 \%$ of the variance in frequency of cutting, with the Rumination, Organization, and Concern over Mistakes (inversely) scales accounting for significant variance independently. Rumination refers to a tendency to obsessively worry about past 
errors, less than perfect performance, and future mistakes. This was the strongest individual predictor of frequency, and has been associated with obsessive-compulsive anxiety [28]. The association between Rumination and cutting is consistent with the findings of Hoff and Muehlenkamp [22], and suggests that a disposition to brood and obsess about one's negative experiences and distress may predispose individuals to self-injury. Hoff and Muehlenkamp [22] also found high Organization associated with frequency of self-injury; however they found high rather than low Concern over Mistakes when assessing mean differences between broader non-clinical self-injury versus no self-injury groups. Organization refers to a tendency to be neat, orderly and disciplined, and is a finding congruent with the association between Conscientiousness and frequency of cutting, while a lack of Concern over Mistakes indicates low distress over the prospect of making errors or having shame over performance failures. The curious outcome of high Rumination and low Concern over Mistakes in the current study suggests it may be valuable to investigate the content of ruminative thought in those who engage in cutting.

Perfectionism was not helpful in predicting duration of cutting, but nearly significant in predicting age of onset of cutting. The significant correlations between age of onset of self-injury and perfectionistic concern over mistakes and need for approval suggest the older individuals are when they start self-injury the more likely they are to be concerned with performance mistakes and need the approval of others when evaluating performance success or setting personal standards. These associations are also congruent with the significant association between age of onset and neuroticism. The older one begins self-injury the more distress is likely to be manifest in the personality of the individual.

\subsection{Summary, Limitations, and Future Directions}

These associations between Big Five traits and self-laceration are somewhat unique in the limited literature describing NSSI and personality as they run counter to some previous findings. In fact, the pattern of correlations reflected in Table 1 describes more complex associations with cutting behaviors than previously reported. In addition to the usual frequency of self-injury, the current investigation added to the knowledge base by assessing duration and age of onset of NSSI, specifically cutting. While this increases the complexity of findings, it also may aid in the understanding of the phenomena of self-injury and highlights the potential for different results depending on specific method of NSSI evaluated. Future studies examining personality constructs might specify particular methods of NSSI as well as duration and age of onset to increase understanding of the behavior and better identify who may be at risk. This investigation was limited by a modest sample size. As such, replication of results is recommended. Secondly, several of the personality trait measures used were abbreviated scales as the results were drawn from a larger study. Additional studies may improve upon the current design by employing longer Five Factor assessment scales.
The implications of these finding are clinically relevant. If different methods of NSSI are associated with different personality profiles and risk factors, treatment may be more successful if modified to target the particular personality profile. For example, treatments for those who cut may benefit from the integration of personality assessments into therapy to address higher levels of Conscientiousness or perfectionism. Awareness of and living more consistently with one's personality and values, or working to accept discrepancies, may decrease distress arising from the discrepancy between values and actions, thus decreasing NSSI. Understanding unique personality profiles associated with NSSI might allow clinicians to provide more specific services.

\section{References}

[1] R. Favazza, Bodies Under Siege: Self-Mutilation and Body Modification in Culture and Psychiatry. John Hopkins U. P.: Baltimore, 1996, pg. 225.

[2] J. Briere and E. Gil, "Self-mutilation in clinical and general population samples: Prevalence, correlates, and functions," American Journal of Orthopsychiatry, vol. 68, pp. 609-620, 1998.

[3] E. D. Klonsky, T.F. Oltmanns, and E. Turkheimer, "Deliberate self-harm in a nonclinical population: Prevalence and psychological correlates," American Journal of Psychiatry, vol. 160, pp. 1501-1508, 2003.

[4] H. Fliege, J. R. Lee, A. Grimm, and B. F. Klapp, "Risk factors and correlates of deliberate self-harm behavior: A systematic review," Journal of Psychosomatic Research, vol. 66, pp. 477-493, 2009.

[5] J. Whitlock, J. Eckenrode, and D. Silverman, "Self-injurious behaviors in a college population," Pediatrics, vol. 117, pp. 1939-1948, 2006

[6] K. L., Gratz, S. D. Conrad, and L. Roemer, "Risk factors for deliberate self-harm among college students," American Journal of Orthopsychiatry, vol. 72, pp. 128-140, 2002.

[7] E. E. Lloyd-Richardson, N. Perrine, L. Dierker, and M. L. Kelley, "Characteristic and functions of non-suicidal self-injury in a community sample of adolescents," Psychological Medicine: A Journal of Research in Psychiatry and the Allied Sciences, vol. 37, pp. 1183-1192, 2007.

[8] S. A. Brown, "Personality and non-suicidal deliberate self-harm: Trait differences among a non-clinical population," Psychiatry Research, vol. 169, pp. 28-32, 2009.

[9] K. L. Croyle and J. Waltz, "Subclinical self-harm: Range of behaviors, extent, and associated characteristics," American Journal of Orthopsychiatry, vol. 77, pp. 332-342, 2007.

[10] K. E. Buckholdt, G. R. Parra, and L. Jobe-Shields, “Emotion regulation as a mediator of the relation between emotion socialization and deliberate self-harm," American Journal of Orthopsychiatry, vol. 79, pp. 482-490, 2009.

[11] C. Zlotnick, M. T. Shea, T. Pearlstein, E. Simpson, E. Costello, and A Begin, "The relationship between dissociative symptoms, alexithymia, impulsivity, sexual abuse, and 
self-mutilation," Comprehensive Psychiatry, vol. 37, pp. 12-16, 1996.

[12] M. Adrian, J. Zeman, C. Erdley, L. Lisa, and L. Sim, “ Emotional dysregulation and interpersonal difficulties as risk factors for nonsuicidal self-injury in adolescent girls," Journal of Abnormal Child Psychology, vol. 39, pp. 389-400, 2011.

[13] M. R. Weierich and M. K. Nock, "Posttraumatic stress symptoms mediate the relation between childhood sexual abuse and nonsuicidal self-injury," Journal of Consulting and Clinical Psychology, vol. 76, pp. 39-44, 2008.

[14] L. Claes, A. Houben, W. Vandereycken, P. Bijttebier, and J. Muehlenkamp, "Brief report: The association between non-suicidal self-injury, self-concept and acquaintance with self-injurious peers in a sample of adolescents," Journal of Adolescence, vol. 33, pp. 775-778, 2010.

[15] L. Claes, W. Vandereycken, and H. Vertommen, H., “ Personality traits in eating disordered patients with and without self-injurious behaviors," Journal of Personality Disorders, vol. 18 , pp. 399-404, 2004.

[16] S. Shea, "Personality characteristics of self-mutilating male prisoners," Journal of Clinical Psychology, vol. 49, pp. 576-585, 1993

[17] A. L. Goldstein, G. L. Flett, C. Wekerle, and A. M. Wall, "Personality, child maltreatment, and substance use: Examining correlates of deliberate self-harm among university students," Canadian Journal of Behavioural Science, vol. 41, pp. 241-251, 2009

[18] V. V. MacLaren and L. A. Best, "Nonsuicidal self-injury, potentially addictive behaviors, and the five factor model in undergraduates," Personality and Individual Differences, vol. 49 , pp. 521-525, 2010.

[19] P. T. Costa and T. A. Widiger, Personality Disorders and the Five-Factor Model of Personality, 2nd ed., Washington, DC: American Psychological Association, 2002.

[20] R. Kotov, W. Gamez, F. Schmidt, and D. Watson, "Linking 'big' personality traits to anxiety, depressive, and substance use disorders: A meta-analysis," Psychological Bulletin, vol. 136, pp. 768-821, 2010.

[21] J. Stoeber and K. Otto, "Positive conceptions of perfectionism: Approaches, evidence, challenges," Personality and Social Psychology Review, vol. 10, pp. 295-319, 2006.

[22] E. R. Hoff and J. J. Muehlenkamp, "Nonsuicidal self-injury in college students: The role of perfectionism and rumination," Suicide and Life-Threatening Behaviors, vol. 39, pp. 576-587, 2009.

[23] R. O. Frost, P. Marten, C. Lahart, and R. Rosenblate, "The dimensions of perfectionism," Cognitive Therapy and Research, vol. 14, pp. 449-468, 1990.

[24] S. Nolen-Hoeksema, "Sex differences in unipolar depression: Evidence and theory," Psychological Bulletin, vol. 101, pp. 259-282, 1987

[25] R. C. O'Connor, S. Rasmussen, and K. Hawton, "Predicting depression, anxiety and self-harm in adolescents: The role of perfectionism and acute life stress," Behaviour Research and Therapy, vol. 48, pp. 52-59, 2010.

[26] K. L. Gratz, "Measurement of deliberate self-harm: Preliminary data on the Deliberate Self-Harm Inventory," Journal of Psychopathology and Behavioral Assessment, vol. 23, pp. 253-263, 2001.

[27] L. R. Goldberg, J. A. Johnson, H. W. Eber, R. Hogan, M. C. Ashton, C. R. Cloninger, and H. C. Gough, "The International Personality Item Pool and the future of public-domain personality measures," Journal of Research in Personality, vol. 40, pp. 84-96, 2006.

[28] R. W. Hill, T. J. Huelsman, R. Furr, J. Kibler, B. B. Vicente, and C. Kennedy, "A new measure of perfectionism: The Perfectionism Inventory," Journal of Personality Assessment, vol. 82, pp. 80-91, 2004.

[29] P. T. Costa and R. R. McCrae, NEO Personality Inventory: Professional Manual, Odessa, FL: Psychological Assessment Resources, 1992. 